\title{
Making Tunnel Barriers (Including Metals) Transparent
}

\begin{abstract}
I. R. Hooper, T. W. Preist, and J. R. Sambles
School of Physics, University of Exeter, Exeter, Devon, EX4 4QL, United Kingdom

(Received 18 January 2006; revised manuscript received 14 June 2006; published 3 August 2006)

The classical "brick wall," which may, according to quantum mechanics, leak via tunneling, is here shown to be completely transparent when appropriate impedance matching media are placed both in front of and behind the "wall." Optical experiments involving beyond-critical-angle-tunnel barriers in the frustrated total internal reflection scheme which mimic quantum mechanical systems provide convincing proof of this remarkable effect. The same mechanism also allows vastly enhanced transmission through unstructured thin metal films without the need for surface wave excitation.
\end{abstract}

DOI: 10.1103/PhysRevLett.97.053902

PACS numbers: 42.25.Bs, 03.65.Xp, 42.50.Xa

Quantum mechanics brought with it the wonderfully nonclassical concept of particle tunneling - the process whereby wave or particles may penetrate through classically impenetrable barriers. Optical physicists had, of course, been aware of tunneling in the somewhat different guise of frustrated total internal reflection (F-TIR) [1,2], whereby evanescent optical fields penetrate across an air gap between two adjacent glass prisms, giving transmission of light beyond the critical angle for total internal reflection. Indeed, the link between quantum mechanics and optics has a long history [3].

Though there is no exact equivalent to a potential for photons, an analogy to quantum mechanics can be considered. The equivalent parameter to the potential is $-n^{2} k_{0}^{2}$, with the equivalent to the total energy being $-k_{x}^{2}=$ $-n_{i}^{2} k_{0}^{2} \sin ^{2} \theta_{i}$ where the subscript $i$ relates to the incident medium, and $k_{0}$ is the wave vector in free space. Ordinary dielectrics have a negative "potential", so they are only tunnel barriers for angles of incidence sufficient to reduce $k_{x}$ such that the "energy " of the incident photon is reduced to below the potential of the tunnel barrier (in other words the light would normally undergo total internal reflection). Materials with a purely imaginary refractive index (ideal metals) have a potential greater than zero and will be tunnel barriers for all angles of incidence (Fig. 1).

Resonant structures which allow enhanced tunneling probabilities are well-known. For example, resonant tunneling is observed in double barrier heterostructure devices (see Chang, Esaki, and Tsu [4]) (incidentally, this can be considered as an electronic analogue of the Fabry-Perot etalon in optics, where the quantum well, sandwiched between the two tunnel barriers, acts a resonant cavity [5]). More recently, it has been shown that in principle $100 \%$ of incident microwaves can be transmitted through a metal meta-material having negative permittivity [6]. In the present study we show that this surprising result is true for all tunnel barriers, whether in the field of optics or, by extension, in any quantum mechanical system. Furthermore, we demonstrate massively enhanced optical transmission through structureless metal films.
Consider an optical wave incident upon a three layer structure of the form $A B A$ such that a central medium allowing only evanescent waves (in quantum mechanics this is a barrier having a purely real potential greater than the energy of the incident wave or particle) is bounded by two identical media, with a third semi-infinite medium on either side. A schematic is shown inset in figure 1, along with the equivalent of the potential diagram for the system (note, this is not equivalent to a Fabry-Perot etalon).

For unitary transmission we require not only that both the wave functions and their derivatives be continuous across all boundaries, but that the amplitude of the incident and transmitted waves be equal. This gives an equation valid for both quantum mechanics and optics:

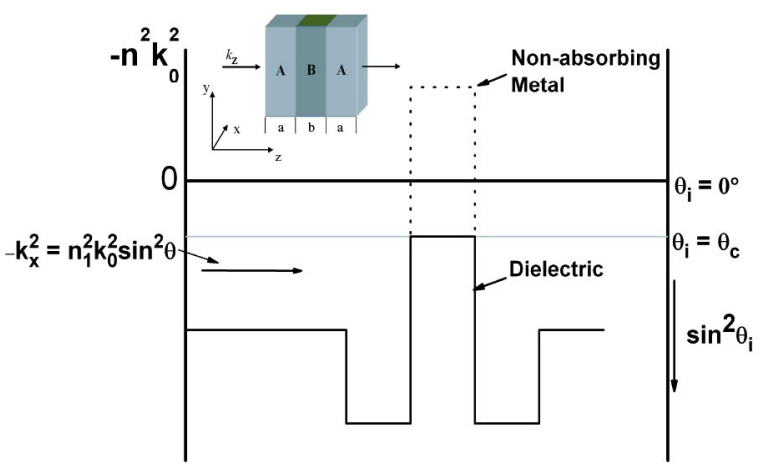

FIG. 1 (color online). The optical equivalent of the potential diagram in quantum mechanics. The analogous quantities are: potential and $-n^{2} k_{0}^{2}$, energy and $-k_{x}^{2}$. Two lines are plotted; the solid line corresponds to a low refractive index central dielectric core surrounded by higher refractive index layers, which are in turn bounded by semi-infinite media of intermediate refractive index, while the dotted line corresponds to an identical system but with an ideal metal central core. The central core in the metal case is a tunnel barrier for all angles of incidence ( $k_{x}$ values), while in the dielectric core case it is only a tunnel barrier for angles of incidence greater than the critical angle for total internal reflection. The inset shows a schematic of the system and the coordinate system used. 


$$
\begin{aligned}
& \left(\frac{K_{z 2}}{K_{z 1}}-\frac{K_{z 1}}{K_{z 2}}\right) 2 \tan \left(k_{z 2} a\right)-\left(\frac{A_{z 3}}{K_{z 1}}+\frac{K_{z 1}}{A_{z 3}}\right) \tanh \left(\alpha_{z 3} b\right) \\
& -\left(\frac{K_{z 2}^{2}}{K_{z 1} A_{z 3}}+\frac{K_{z 1} A_{z 3}}{K_{z 2}^{2}}\right) \tan ^{2}\left(k_{z 2} a\right) \tanh \left(\alpha_{z 3} b\right)=0 ;
\end{aligned}
$$

here $a$ and $b$ are the thicknesses of layers $A$ and $B$ respectively, $K_{z i}=\frac{k_{z i}}{\varepsilon_{i}}$ for transverse magnetic (TM) polarized light or $K_{z i}=\frac{k_{z i}}{\mu_{i}}$ for transverse electric (TE) polarized light and $K_{z 3}=i A_{z 3}$, with $k_{z 3}=i \alpha_{z 3}$. Subscript $i=1$ corresponds to the incident or exit media, $i=2$ medium $A$ and $i=3$ medium $B$ (the tunnel barrier). $k_{z i}$ is the wave vector normal to the structure, and $\varepsilon_{i}$ and $\mu_{i}$ are the relative permittivities and permeabilities. For TE polarized light, if $\mu_{1}=\mu_{2}=\mu_{3}=1$ this equation becomes identical to the quantum mechanical one, while the same is true for TM polarized light when $\varepsilon_{1}=\varepsilon_{2}=\varepsilon_{3}=1$. TE polarized light can be readily used to simulate quantum mechanical systems, since many materials are nonmagnetic $(\mu=1)$, whereas the equivalent condition for TM polarized light is not generally possible. Therefore, since we wish to show that unitary transmission can occur for any quantum mechanical system, TE polarized light is first examined.

Consider the physical meaning of solutions to Eq. (1) for a simple optical system. A dielectric layer of thickness $80 \mathrm{~nm}$ and refractive index of 5 has a tunnel barrier with a purely imaginary refractive index of $5 i$ at its center. Light is normally incident and the transmission as a function of frequency, and thickness of the tunnel barrier, is modeled using multilayer optics theory. The results are shown in Fig. 2(a). For no tunnel barrier unitary transmission is evident at approximately $0.38 \times 10^{15} \mathrm{~Hz}$ and $0.56 \times$ $10^{15} \mathrm{~Hz}$. This occurs when the dielectric slab resonantly satisfies $\lambda=2 \mathrm{nd} / \mathrm{m}$ where $n$ is the refractive index of the dielectric slab, $d$ is its thickness, and $m$ is an integer. The two modes shown in Fig. 2(a) are the second and third order modes $(m=2,3)$. These are Fabry-Perot-type resonances, though not of the typical etalon type as they are resonant in the $H$ field rather than the $E$ field. Increasing the thickness of the tunnel barrier results in the two resonances converging to a frequency of approximately $0.47 \times$ $10^{15} \mathrm{~Hz}$, where unitary transmission still occurs up to a maximum tunnel barrier thickness of $\sim 33 \mathrm{~nm}$. A different choice of refractive indices (depth and height of the potential wells and/or barriers) and of the incident angle (energy of the incident particle) gives different limits on the maximum tunnel barrier thickness still allowing unitary transmission.

The time averaged $E$ and $H$ fields for 3 points in Fig. 2(a) are shown in Fig. 2(b)-2(d) for the two modes with a $5 \mathrm{~nm}$ thick tunnel barrier, and for the single mode with a $33 \mathrm{~nm}$ tunnel barrier. Odd order modes [e.g., Fig. 2(c)] have a minimum in the $E$ field at the center of the structure, and hence it is unsurprising that the inclusion of a thin tunnel barrier has little influence (although it may
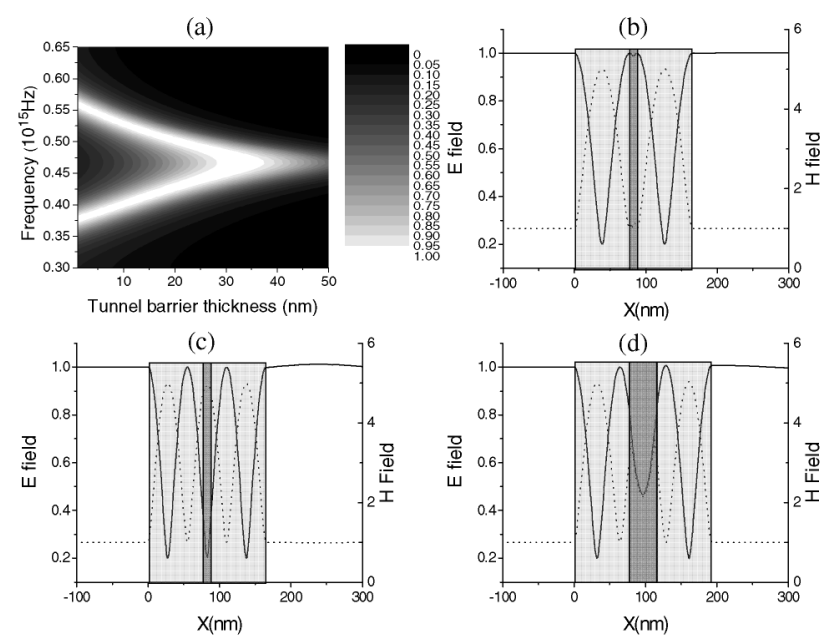

FIG. 2. (a) Normal incidence transmission as a function of frequency and tunnel barrier thickness through a tunnel barrier with a purely imaginary refractive index of 5, surrounded symmetrically by two $80 \mathrm{~nm}$ thick dielectric layers having a purely real refractive index of 5 in vacuum. (b), (c), (d) $E$ (solid lines) and $H$ fields (dotted lines) of the modes at (b) $f=0.38 \times$ $10^{15} \mathrm{~Hz}$ and a thickness of $5 \mathrm{~nm}$, (c) $f=0.56 \times 10^{15} \mathrm{~Hz}$ and a thickness of $5 \mathrm{~nm}$, and (d) $f=0.47 \times 10^{15} \mathrm{~Hz}$ and a thickness of $33 \mathrm{~nm}$.

still be expected that unitary transmission would not be possible as soon as any tunnel barrier is added). However, the even order modes have a maximum of $E$ field at the tunnel barrier and yet unitary transmission still occurs. Figure 2(d) for a larger thickness tunnel barrier confirms that any simple concept involving low $E$ fields at the barrier being the origin of the unitary transmission is incorrect. The real reason for the unitary transmission is that the combination of interface reflections interfere such that the reflection from the structure is zero; with nonabsorbing media the transmission must then be unitary. In other words, the dielectric layer (wells) on either side of the barrier act as perfect impedance matching structures.

Consider a simple realization of such a system. In Ref. [7] the tunnel barrier used in their experimental investigation was a meta-material with a permittivity consisting of a negative real part and zero imaginary part. Though this is a tunnel barrier in that the wave vector in the meta-material is purely imaginary, a far simpler system is one exhibiting F-TIR. In this case the refractive indices are all real and positive, and it is easily realizable.

Typically in an F-TIR system, two prisms are brought very close to each other with light incident beyond the critical angle, so that the gap between the prisms acts as a tunnel barrier. By introducing high refractive index layers on either side of the barrier the necessary "potential wells " are formed creating a potential diagram similar to that shown in Fig. 1.

In practice, two $45^{\circ}$ silica prisms were coated on their hypotenuse faces with thin layers of $\mathrm{ZnS}$ via evaporation 
under vacuum. (The thickness of the $\mathrm{ZnS}$ was approximately known from a quartz film thickness monitor). These were then clamped together to produce a very small air gap between them and wavelength dependent transmission data were obtained for various angles of incidence and air gap widths for both TM and TE polarized light. A schematic is shown in the inset of Fig. 3(a). By fitting the multiple data sets simultaneously to multilayer optics theory, while maintaining fixed layer thicknesses between data sets, the thickness of both $\mathrm{ZnS}$ layers and the dispersion of their complex refractive indices throughout the wavelength range were obtained, though since the $\mathrm{ZnS}$ layer thickness was approximately known tight bounds were imposed upon it. Using these parameters with Eq. (1) and for an incident internal angle (measured with respect to the normal) of $68.2^{\circ}$ the air gap thickness allowing unitary transmission is determined as $133.7 \mathrm{~nm}$ at a wavelength of $700 \mathrm{~nm}$. The air gap was adjusted to be as close to this value as possible, and wavelength dependent TE polarized transmission data obtained and fitted to theory using the same parameter values obtained from the previous multiple data set fitting process, allowing only the thickness of the air gap to vary. This fitting gave an air gap thickness of $131 \mathrm{~nm}$, very close to the value of $133.7 \mathrm{~nm}$ calculated from Eq. (1).

The resultant data, corrected to remove the reflections at the external faces of the prisms, together with the multilayer optics prediction, is shown in Fig. 3(a). Clearly the transmission peak at $700 \mathrm{~nm}$ is approximately $85 \%$. This small deviation from $100 \%$ is due to absorption in the $\mathrm{ZnS}$ layer which is not accounted for in the solution to Eq. (1) where the refractive index of the $\mathrm{ZnS}$ is assumed to be purely real. Also plotted in Fig. 3(a) is the transmission expected if no $\mathrm{ZnS}$ layers were present (the traditional FTIR device). This clearly demonstrates the remarkable increase in transmission obtained by simply introducing the $\mathrm{ZnS}$ layers.

Though the special case of unitary transmission is the most dramatic of situations possible, often it is not possible to realize a system which will allow this due to the limits on the material parameters available. However, vastly increased transmission is still possible, even when absorption is significantly greater than in the previous F-TIR experiment. This can be simply demonstrated by substituting a metal film as the tunnel barrier.

There has been considerable interest in recent years in increased optical transmission through structured thin metal films via the excitation of surface plasmon polaritons (SPPs) [7-11]. A metal film acts as a tunnel barrier for all angles of incidence because the imaginary part of its refractive index is much larger than the real part. However, metal films are not ideal tunnel barriers since absorption occurs due to the finite real part of the refractive index. It is often assumed that the low transmission through metal films is due to this absorption, but this is not the case; it is predominantly due to the large impedance mismatch at the interfaces of the bounding dielectrics with the metal film giving very strong reflections, with the fields penetrating the metal film thus being very small. The quantum analogy is a very high tunnel barrier with a slightly complex potential. Now, if the impedances are ideally matched the only loss in intensity in the transmission is due to the
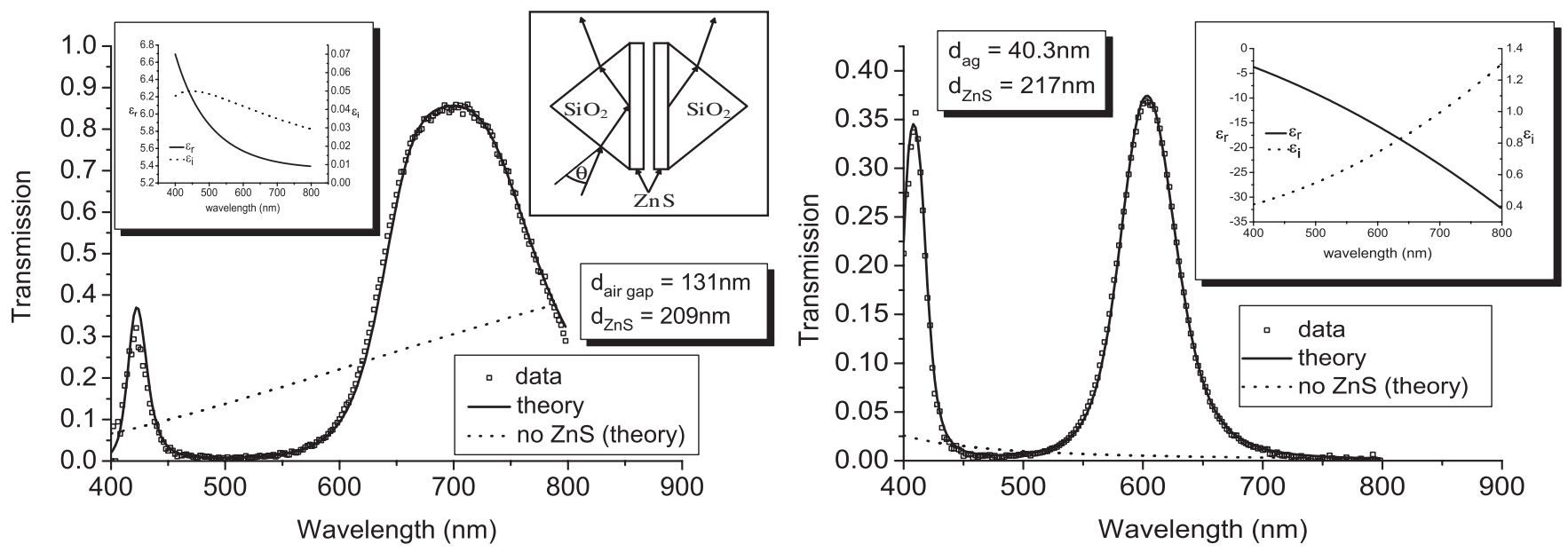

FIG. 3. (a) TE polarized transmission from the system shown inset (adjusted to account for reflections from the external faces of the prisms). The incident internal angle is $68.2^{\circ}$. By fitting multiple data sets for different angles of incidence for both TE and TM polarizations to multilayer optics theory the parameters of the system are determined. These are given in the figure. The index dispersion of $\mathrm{ZnS}$ obtained from the fitting is shown inset, agreeing well with published values. Also plotted is the model best fit, as well as the transmission predicted if there were no $\mathrm{ZnS}$ layers present. (b) Similar data sets to those in (a) except that the air gap has been replaced by a silver film and the incident internal angle is $71.2^{\circ}$. The system parameters have been obtained via fitting data to theory in the same way as for (a), using the index dispersion of $\mathrm{ZnS}$ as obtained in (a). The dispersion of the silver dielectric function as well as the system parameters obtained from the fitting process are shown in the inset. 
absorption within the metal film. However, the imaginary part of the refractive index of a metal in the optical region of the spectrum is very high. Therefore, to perfectly match the impedances (for normal incidence radiation) with layers of dielectric either side of the metal is not practicable due to the necessarily high refractive index dielectric required. However, very strongly enhanced transmission relative to that expected for a film not bounded by the higher refractive index layers is still possible (typically $>10$ times for the majority of situations).

Though metal films are tunnel barriers for all angles of incidence, prisms on the input and exit faces allow a larger range of incident angles to be studied. Increasing the angle of incidence reduces the normal component of the incident wave vector, and as this tends to zero it allows for solutions to Eq. (1) without the need for such high index well layers. Thus, to investigate the enhanced tunneling through a metal film a similar structure to that utilized in the F-TIR system described above was used [inset of Fig. 3(a)], with the air gap replaced by a silver film (the system was manufactured by evaporating the $\mathrm{ZnS}$ and silver in alternate layers under vacuum). By taking wavelength dependent transmission data for both TE and TM polarized light for various angles of incidence the thickness of the $\mathrm{ZnS}$ layers, and the thickness and wavelength dependent dielectric function of the silver, were, as before, found by fitting the data with multilayer optics theory (the thickness values were restricted to tight bounds as they were known approximately through monitoring during the deposition process). The dielectric function obtained for the silver is very similar to that given in the literature [12], and the dielectric function of the $\mathrm{ZnS}$ is the same as for the films used in the F-TIR experiment. The data corresponding to an internal incident angle of $71.2^{\circ}$ is shown in Fig. 3(b), as is the corresponding theoretical fit obtained from multilayer optics theory. Also shown is a model curve for the transmission through an identical system without $\mathrm{ZnS}$ layers.

The angle of incidence for Fig. 3(b) does not correspond to the data set that shows the largest enhancement in transmission over that of the equivalent system without $\mathrm{ZnS}$ layers. Indeed, in the data presented in Fig. 3(b) the enhancement at the peak around $600 \mathrm{~nm}$ is $\sim 80$ times, substantially in excess of that found in most SPP enhanced transmission configurations. However, the enhanced transmission in this case works for both TE and TM polarized light over a wide range of incident angles, unlike the case of SPP enhanced transmission, and furthermore the metal film is structureless.

In this Letter we have demonstrated that there are configurations which allow unitary transmissions through tunnel barriers, which can be simply realized by placing wells of the appropriate depth and thickness both before and after the barrier. This is true for all quantum mechanical systems, and has been demonstrated here through the use of a TE polarized light transmission study of frustrated total internal reflection. Enhanced transmission of electromagnetic radiation through metal mirrors has also been shown to be possible with no need for elaborate structuring. Though the experiments presented here are photonic in nature, it is important to note that this is predominantly for ease of experimentation. These results may have far reaching implications for any tunneling situation, and experiments demonstrating the effect in quantum systems would be of great interest.

[1] E. E. Hall, Phys. Rev. 15, 73 (1902).

[2] S. Zhu, A. W. Yu, D. Hawley, and R. Roy, Am. J. Phys. 54, 601 (1986).

[3] Th. Martin and R. Landauer, Phys. Rev. A 45, 2611 (1992).

[4] L. L. Chang, L. Esaki, and R. Tsu, Appl. Phys. Lett. 24, 593 (1974).

[5] L. Eaves, in Analogies in Optics and Micro Electronics, edited by W. van Haeringen and D. Lenstra (Kluwer Academic Publishers, Dordrecht, 1990).

[6] L. Zhou, W. J. Wen, C. T. Chan, and P. Sheng, Phys. Rev. Lett. 94, 243905 (2005).

[7] H. Raether, Surface Plasmonson Smooth and Rough Gratings (Springer-Verlag, Berlin, 1988).

[8] R. Dragila, B. Luther-Davies, and S. Vukovic, Phys. Rev. Lett. 55, 1117 (1985).

[9] T. W. Ebbesen, H. J. Lezec, H.F. Ghaemi, T. Thio, and P. A. Wolff, Nature (London) 391, 667 (1998).

[10] W. L. Barnes, W. A. Murray, J. Dintinger, E. Deveaux, and T. W. Ebbesen, Phys. Rev. Lett. 92, 107401 (2004).

[11] N. Bonod, S. Enoch, L. Li, P. Evgeny, and M. Nevière, Opt. Express 11, 482 (2003).

[12] Handbook of Optical Constants of Solids, edited by E. D. Palik (Academic, New York, 1985). 